\section{P-60 OPTIMISING HOW DIFFERENT HOSPICE TEAMS USE AN ELECTRONIC PATIENT RECORD (SYSTMONE): THE HOLISTIC TEMPLATE}

Gemma Miller, Stacey Hubber, Luke Feathers. LOROS Hospice, Leicester, UK

\subsection{6/bmjspcare-2016-001245.84}

Background Our organisation has been using SystmOne as our electronic patient record since 2012. In 2014, a comprehensive review of how different teams were using the system identified that data was inconsistently recorded and key information was difficult to locate, proving frustrating and time-consuming.

Our goal was to standardise data entry, to improve accuracy and reduce duplication of data within the patient record.

Aims To improve the use of an electronic patient record so that clinicians can view previous entries more clearly, reduce duplication, avoid missing data and facilitate reporting measures to enhance patient care.

Methods The organisation worked as a collective to create a bespoke and comprehensible holistic template that could meet the needs of all teams (excluding Lymphoedema). The new template included sections around 'patient insight and information needs', 'carer needs' and 'family bereavement issues', responding to the suggested National Dataset Outcome Measures (MDS) and a local audit on bereavement needs.

An agreed 'Go live' date was publicised, training was provided and staff supported through the transition.

Results

- Improved quality of the shared patient record

- Key information more accessible

- Comprehensive demographic and geographic information as per MDS requirements

- Provides detailed clinical information to the senior management team and the board to make strategic decisions on the future development of the care provision

- Helps to deliver coordinated care across the multi-disciplinary teams

- Integrated advance care planning via the use of agreed coding with external health and social care providers

- New way of recording patient measure (Phase of illness, Australian Karnofsky Performance Status and Integrated Palliative Outcome Score) supporting clinical staff to provide more personalised care

- New assessment domains allowing key areas to be covered

- Incorporation of built-in views to avoid duplication and to identify missing information.

Conclusion The new holistic template is viewed as an example of best practice and our organisation is now sharing the template with other providers.

\section{P-61 INCORPORATING THE INTEGRATED PALLIATIVE OUTCOME SCORE IN TPP SYSTMONE}

Gemma Miller, Luke Feathers, Stacey Hubber. LOROS Hospice, Leicester, UK

\subsection{6/bmjspcare-2016-001245.85}

Background During 2015, our organisation started using the Integrated Palliative Outcome Score (IPOS) questionnaire in the Day Therapy environment as part of a three-month pilot. Efforts were made to manage the IPOS data within SystmOne, but the lack of specific read-coding prevented the development of a template. Various other solutions including bespoke IT systems were investigated.

In 2016, our organisation's Clinical Systems Lead worked with TPP (creators of SystmOne) to develop unique local (Y) codes for the IPOS, which has enabled data to be directly captured and compared within SystmOne.

Aim To record IPOS data directly within SystmOne, to help deliver and evaluate high quality patient care as set out by the Cicely Saunders Institute (OACC Project).

Methods After IPOS codes were published, the Clinical Systems Lead worked collaboratively with other professionals, both internal and external, to create a functional SystmOne IPOS clinical template, which could be used across all clinical services and shared with other Healthcare organisations.

Results

- Able to capture individual patient symptoms and concerns, and display the outcomes over time, for example at multidisciplinary meetings

- Outcome measures can be used for clinical care, audit and research; enhancing information sharing

- Enable the improvement of individual patient care and easier evaluation of outcomes across different care services

- Enable compliance with the new national dataset for specialist palliative care

- Support informed strategic decisions around service outcomes and developments, including training needs for staff

- Electronic record of outcome measures enables improved access to accurate patient data

- Changes in patients' health status can be monitored and themes identified.

Conclusion Collaboration between palliative organisations and direct engagement with the developers of an electronic care record has enabled better recording and data handling for patient outcome measures within the existing clinical system. This work is being shared nationally.

\section{P-62 EPACCS IMPLEMENTATION: ACHIEVING CLINICAL ENGAGEMENT THROUGH BOTTOM-UP TRANSFORMATIONAL CHANGE}

Louise Price. St Barnabas Lincolnshire Hospice, Lincoln, UK

10.1136/bmjspcare-2016-001245.86

Electronic Palliative Care Co-ordination Systems (EPaCCS) are an electronic means of communication and coordination at endof-life. Arguably implementation of EPaCCS should now be a high priority for all, it will contribute significantly to achieving Ambitions 2015-2020, it is an outcome for end-of-life care in NHS England's Sustainability Transformational Plans (STPs) and supports general practice now that CQC are inspecting against the end-of-life domain.

Information technology (IT) enables the EPaCCS outcomes and anticipated benefits to be achieved; but it is not in isolation. Implementation requires multiple interdependent factors to ensure utilisation of EPaCCS and realise patient benefits. These include, but are not isolated to, engagement and training, change 
of clinical behaviours and practice, and identifying palliative patients.

Following initial scoping the IT enabler of choice, My RightCare (MRC) was identified for the locality and was subsequently grant funded by the NHS England Nursing Technology Fund. Unlike other solutions MRC creates a patient-centric unified care plan, specifically being clinician friendly, with functionalities such as auto-population and flagging in your own system. Created care plans, are then shared (with consent) across all health care IT systems; primary and secondary care, including urgent care; ambulance and NHS 111 services. This coupled with developing a suite of tools to support GPs and provider colleagues to utilise as they choose, has supported, engaged, and empowered them to improve coordination of end of life care in the county and create a social movement on the frontline.

We would like to share the experience of a large rural county in achieving clinical engagement through bottom-up transformational change. With a small budget and little authority, but with innovative technology and a different approach we are implementing EPaCCS in our locality. And there are no project plans!

\section{P-63 IMPLEMENTATION OF A PALLIATIVE CARE ELECTRONIC PATIENT RECORD SHARED WITH GPS, DNS AND THE OUT OF HOURS SERVICE}

${ }^{1}$ Susan Salt, ${ }^{1}$ Marie Bonnis, ${ }^{1}$ Nicky Parkes, 'Debra Green, ${ }^{1}$ Carol Wylde, ${ }^{1}$ Victoria Murphy, ${ }^{2}$ Bridget Lord. 'Trinity Hospice, Blackpool, UK; ${ }^{2}$ Blackpool Teaching Hospitals NHS Foundation Trust

10.1136/bmjspcare-2016-001245.87

\section{Aims of the Project}

1. To move to an electronic patient record across all clinical departments accessible to local community services enabling access to real time information about a patient's care and condition shared across the hospice, general practice, district nursing services and out-of-hours service

2. Enable hospice staff to remotely access a patient's up-to-date record enabling advice given and care delivered to be recorded without having to find a set of case notes or returning to base

3. Reduced amount of telephone and fax traffic between primary care and the hospice around clarifying care, medication and other key factors such as known allergies

4. Saving time on administration and record keeping to enable more clinical time.

Over a period of nine months using a change management approach an instance of the electronic patient record (EMIS) was developed for each clinical team, based on a common holistic assessment. At all stages staff engagement and communication was a high priority.

Roll out was staggered over a one month period with GO LIVE starting with the smaller day time services, to iron out any teething problems, using the lessons learnt in the bigger 24-hour services including both an adult and children's in-patient unit. (For patient confidentiality reasons the bereavement service was excluded from the whole process.)

\section{Outcomes}

1. Having completed level 2 of the information governance toolkit and compliance with information governance requirements locally all healthcare professionals in the community over 24 hours are able to access real time information about all patients known to the specialist palliative care service

2. The specialist palliative care service can access the records of patients referred to them and those where advice is requested

3. Accuracy of medication histories, compliance and allergy recording has improved

4. The amount of time lost by clinical staff making phone calls has reduced by a third.

\section{P-64 THE CHALLENGES INVOLVED IN EMBEDDING THE PILOT PALLIATIVE CARE DATASET WITHIN AN ELECTRONIC PATIENT RECORD (SYSTMONE)}

Sarah Wells. Marie Curie Hospice, West Midlands, Solihull, UK

10.1136/bmjspcare-2016-001245.88

Background Our hospice was accepted as a pilot site for the Pilot Palliative Care Data set by Public Health England (PHE) in July 2015. The hospice was paper-lite using the electronic patient record system-SystmOne (S1).

Aim To embed any future dataset within S1 to ensure that data was captured within standard working practices enhance patient care as well as improve reporting of patient outcomes.

Methods There were three distinct elements to this pilot project:

1. Changing culture and the language used by staff The pilot dataset included measures of performance status (Karnofsky), complexity (Phase of illness) and patient outcome measures. Champions identified within each staff discipline and education was provided on all measures.

2. Configuration of S1 for data collection Extensive reconfiguration of S1 was required involving incorporation of the concept of "Spells of care", creation of templates and protocols

3. Creation of reporting tools

The granularity of data for the dataset required a different method of reporting to that used previously. This enabled access to a huge amount of raw data but has required an external data analyst company to produce the required reports

Results Seven months after commencing the pilot:

1. Changing the culture and language is taking time. This requires a stepwise approach but gradually these elements are becoming embedded across all sectors

2. SystmOne has now been configured to include all the data items required.

3. Reports were submitted to PHE but are now being fine-tuned to meet our needs.

Interpretation and conclusions To embed a new dataset within an EPR including all of the above elements takes longer than the six months anticipated. Although the palliative care dataset will not be taken forward, the elements of it are proving to be valuable resources that we feel will improve patient care and enable us to evidence the quality of care provided.

\section{P-65 DEVELOPING DOCUMENTATION FOR END-OF-LIFE CARE}

Joy Farquharson. St Andrew's Hospice, Airdrie, UK

10.1136/bmjspcare-2016-001245.89 